\title{
Feature Selection by Relevance Analysis for Abandoned Object Classification
}

\author{
Johanna Carvajal-González, \\ Andrés M. Álvarez-Meza, and German Castellanos-Domínguez
}

Signal Processing and Recognition Group, Universidad Nacional de Colombia, Manizales, Colombia

\{jpcarvajalg, amalvarezme, cgcastellanosd\}@unal.edu.co

\begin{abstract}
A methodology to classify abandoned objects in video surveillance environments is proposed. Our aim is to determine a set of relevant features that properly describes the main patterns of the objects. Assuming that the abandoned object was previously detected by a visual surveillance framework, a preprocessing stage to segment the region of interest from a given detected object is also presented. Then, some geometric and Hu's moments features are estimated. Moreover, a relevance analysis is employed to identify which features reveal the major variability of the input space to discriminate among different objects. Attained results over a real-world video surveillance dataset show how our approach is able to select a subset of features for achieving stable classification performance. Our approach seems to be a good alternative to support the development of automated video surveillance systems.
\end{abstract}

Keywords: Video surveillance, abandoned object classification, feature relevance analysis.

\section{Introduction}

Video surveillance has always been a vital component of security systems. High-level video interpretation tasks related to surveillance are usually completely performed by human operators, who have to process large amounts of visual information presented to them through one or more monitor [1. Recent years have seen a growing demand of automated video surveillance systems for public safety and security enhancement [2]. Unattended objects in public premises such as airports, terminal bus and train stations include mainly bags and trolleys. Bags are a threat for these places because they can be used as a means of terrorist attack, especially for bombs 3 . Trolleys are left abandoned, and they are not a threat for the secured areas, causing nuisance alarms. In 4 address the problem of determining which feature estimation technique proves more useful for accurate object classification in a video surveillance context (scale invariant image transformed key points vs. geometric primitive features), concluding that the classifier based on the set of statistics of geometric primitive features achieved the highest recognition accuracy, and the lowest false alarm rate. However, they need a high dimensional feature set to represent the objects, and no model for reducing dimensionality is 
used. Another work attempts in the classification of different types of bags [5], selecting the optimal feature subset by iterative training a Support Vector Machines classifier with all the possible combinations of the input feature space. Nonetheless, it leads in an exhaustive and complex procedure. On the other hand, besides the geometric and shape features, both based on edge detection or contours, Hu's invariant moments have been extensively applied to image pattern recognition, image registration, and image reconstruction [6]. In this sense, it is necessary to identify a set of features that suitable highlights discriminating patterns to classify abandoned objects, achieving a stable performance with low complexity in automated video surveillance systems.

In this work, a methodology to classify abandoned objects in video surveillance environments is proposed. We aim to identify a set of relevant features that properly highlights discriminating patterns to classify abandoned objects, in order to support the development of automated video surveillance systems. It is important to note that our approach assumes that the abandoned object was previously detected by a visual surveillance framework. Besides, a preprocessing stage to segment the region of interest from a given detected object is also described. Then, some geometric and Hu's moments features are estimated. Moreover, a relevance analysis is employed to select which features reveal the major variability of the input space to discriminate among different objects. Some previous works 78 have shown how analyzing the amount of variability that each feature provides to the studied process, allows to identify discriminative subsets of features for enhancing the classification performance while diminishing the computational complexity. The methodology is experimentally verified on a real-world video surveillance data set, which aim to differentiate among bags, trolleys, and humans in public buildings under potential terrorism attacks.

The remainder of this work is organized as follows. In section 2 the proposed methodology is described. In section 3. the experimental conditions and results are presented. Finally, in sections 4 and 5 we discuss and conclude about the attained results.

\section{Abandoned Object Classification}

The main structure of a video surveillance system involves detection, tracking, and recognition of objects. Considering the objects involved in a video surveillance environment can be either moving (e.g., people, groups of people etc.), or abandoned (e.g., bags, trolleys, etc.) [9], our aim is to develop a methodology that automatically classifies abandoned objects, finding a set of relevant features that highlight appropriate patterns. For such purpose, we assume that the abandoned object was detected by a visual surveillance framework, using for example, a background subtraction method [10]. Following, the main sketch of our approach is described.

\subsection{Image Preprocessing}

Given an image $I_{\text {in }}$ of a bounding box that includes an abandoned object, which is left unattended for a specific period of time, it is necessary to develop some 
previous preprocessing steps that allow to properly segment and characterize the object. Regarding, $I_{i n}$ is transformed to gray-scale color space $\left(I_{\text {gray }}\right)$. After that, the image is smoothed using a filter to reduce possible image noise components. Then, an adaptive thresholding operation is performed, obtaining a binary image $I_{b}$, which encoded the region of interest of the bounding box. This image is taken as the input to the contour detection method, needed to segment the object of interest, removing the presented background. It is important to note that the segmentation is based on the assumption that the bigger contour corresponds to the object of interest, which is drawn as $I_{\text {mask }}$. Finally, the object is cropped using this mask and the segmented unknown object of interest is obtained.

\section{$2.2 \quad$ Feature Estimation}

Let $I_{\text {mask }}$ be a binary image containing the region of interest, in order to describe properly the shape of an abandoned object, some geometric features are estimated to infer the shape properties of the studied objects. These geometric features can contain corners, circles, lines, fitting ellipses, bounding box properties, among others [5411. Moreover, the moment invariants (Hu's Moments) are also considered, which have been proven to be the adequate measures for tracing patterns regarding the images' translation, scaling and rotation, under the assumption of images with continuous functions and noise-free [6]. A full list of the considered features is illustrated in Table 1.

Table 1. Considered features for abandoned object classification

\begin{tabular}{|l|l|l|l|}
\hline Lines & Corners & Ellipses & Other Features \\
\hline - Percentage of lines: diagonal, & - Total of corners. & - Fitting ellipse & - Bounding box \\
horizontal and vertical. & & aspect ratio. \\
- Vertical lines: percentage of lines & - Percentage of corners: left, \\
placed at the left, right and in the center. & - Fitting ellipse & - Hu's moments. \\
- Horizontal lines: percentage of lines placed & - Horizontal and vertical \\
at the top, bottom and in the middle. & standard deviation. & & \\
\hline
\end{tabular}

\subsection{Feature Selection Based on Relevance Analysis}

Principal Component Analysis (PCA) is a statistical technique applied to find out a low-dimensional representation of the original feature space, searching for directions with greater variance to project the data. Although PCA is commonly used as a feature extraction method, it can be useful to select a relevant subset of the original features that better represent the studied process [7/8]. Given a set of features $\left(\boldsymbol{\xi}_{k}: k=1, \ldots, p\right)$ corresponding to each column of the input data matrix $\mathbf{X} \in \Re^{n \times p}$ (e.g., a set of $p$ features describing $n$ abandoned objects), the relevance of $\boldsymbol{\xi}_{k}$ can be identified looking at the values of the relevance vector $\boldsymbol{\rho} \in \Re^{p \times 1}$. Based on a variability analysis as in traditional PCA, $\boldsymbol{\rho}$ is computed as $\boldsymbol{\rho}=\sum_{j=1}^{m}\left|\lambda_{j} \mathbf{v}_{j}\right|$, where $\lambda_{j}$ and $\mathbf{v}_{j}$ are the eigenvalues and eigenvectors of the covariance matrix $\mathbf{S} \in \Re^{p \times p}$ computed as $\mathbf{S}=\mathbf{X}^{\top} \mathbf{X}$. 
Therefore, the main assumption is that the largest values of $\rho_{k}$ point out to the best input attributes, since they exhibit higher overall correlations with principal components. Traditionally, the $m$ value is fixed as the number of dimensions needed to conserve a percentage of the input data variability. Regarding, the eigenvalues $\lambda_{j}$ can be analyzed to infer $m$ [7]. Hence, it is expected that a subset containing the most relevant input features according to $\rho$ are enough to achieve a reliable classification performance. The proposed methodology for abandoned object classification can be summarized as in Fig. 1.

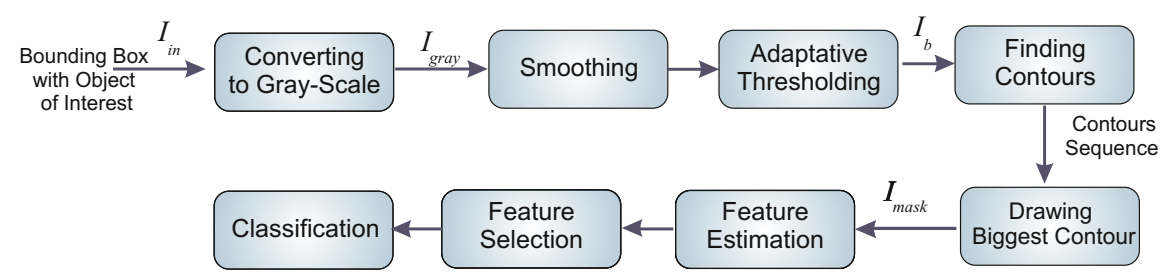

Fig. 1. Abandoned object classification: General Scheme

\section{Experiments and Results}

We aim to test the capability of our approach to identify a relevant set of features for abandoned object classification in video surveillance environments. In this sense, a set of footages from a real-world video surveillance data set provided by the partner company is considered. The footages were recorded in airports, train stations and other public buildings under potential terrorism attacks. The objects of interest were manually cropped, choosing bags, trolleys and people. Furthermore, the studied dataset is completed with some web images to achieve a representative sample. The images were taken under different conditions, varying the lighting, the pose, and the resolution. Therefore, 82 images of bags, 96 images of trolleys, and 70 images of people (one person per image) are considered. All images are stored as 24-bit JPEG images using the RGB color space ( 8 bits per color channel). Fig. 2 shows some images of the tested data set.

Then, a preprocessing stage is performed over the samples in order to detect the region of interest from each image, as mentioned in section 2.1. Particularly, a gaussian filter is used to remove noise from the original bounding box, using a $3 \times 3$ window, and a kernel-width of 0.95 . The adaptive threshold is calculated in a pixel by pixel basis, by computing a weighted average (according to a Gaussian Function of their distance from that center point) of a $151 \times 151$ region around each pixel location minus 10. The above mentioned free parameters were empirically fixed. Moreover, the geometric features and the Hu's moments described in section 2.2 are computed over each detected object. Thus, an input space with 248 samples, 27 features, and 3 classes is obtained $\left(\mathbf{X} \in \Re^{248 \times 27}\right)$. The Open Computer Vision library (OpenCV) was used to calculate the feature set. Besides, to identify the discriminant feature capability, a feature selection 
stage based on relevance analysis is performed according to section 2.3. Here, a 10-fold cross validation scheme is made to generate a mean weighted vector $\overline{\boldsymbol{\rho}}$, with $\overline{\boldsymbol{\rho}_{k}}=1 / 10 \sum_{i=1}^{10} \boldsymbol{\rho}_{k i}$, being $\boldsymbol{\rho}_{k i}$ the weight of relevance of the $k$-th feature in the $i$-th fold. The number of considered eigenvectors and eigenvalues $m$ to computed $\boldsymbol{\rho}_{k i}$ is estimated looking for a $95 \%$ of retained input data variability.

A soft-margin Support Vector Machines (SVM) classifier and a $k$-nearest neighbors classifier (KNN) are trained. We generate a curve of performance adding one by one the features, which are sorted according to $\bar{\rho}$. For a given subset, the optimum working point is fixed using a 10 -fold cross validation to fix the tradeoff parameter $C \in \Re^{+}$between the solution size and training errors, and the gaussian kernel band-width $\sigma$ value in SVM, and to choose the number of neighbors in KNN. Fig. 3 shows some image preprocessing results. Finally, Fig. 4 presents the classification performances.

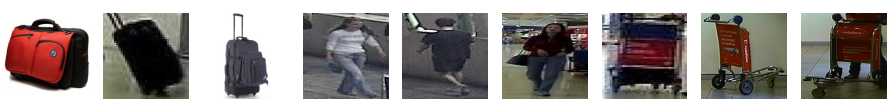

Fig. 2. Database image examples

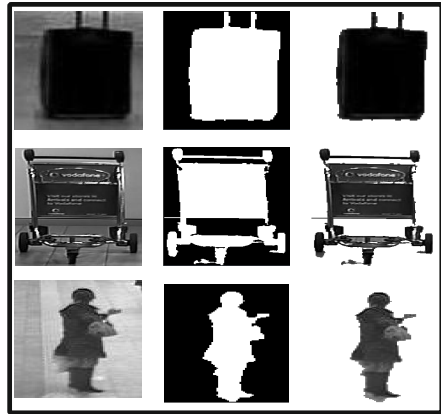

(a)

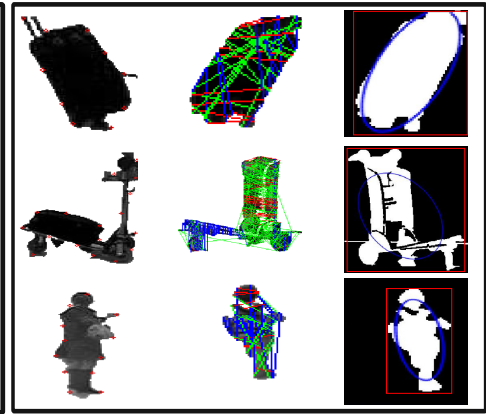

(b)

Fig. 3. Image preprocessing examples results. (a) Segmentation: $I_{\text {gray }}$ at the left side, $I_{\text {mask }}$ in the center and $I_{\text {segm }}$ at the right side. (b) Geometric features: Corners at the left side, lines in the center and fitting shapes at the right side.

\section{Discussion}

From Fig. 3(a) it can be seen how the proposed preprocessing scheme (section 2.1) is able to segment the object of interest, given the bounding box by a video surveillance framework for abandoned object detection. Hence, the attained segmented images are useful to characterize the shape properties of the object. Now, Fig. 3(b) exhibits how some of the proposed geometric features seem to be suitable to find discriminant patterns among objects. 


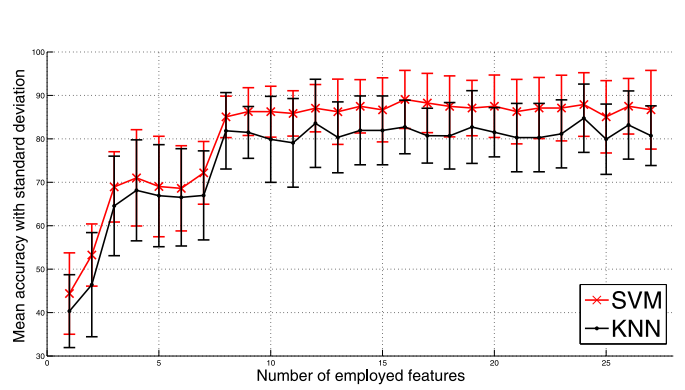

(a)

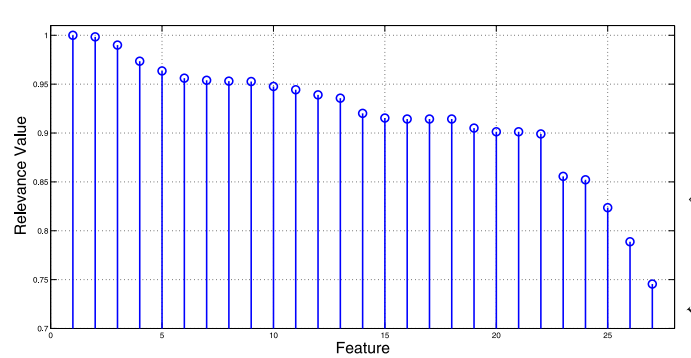

(c)

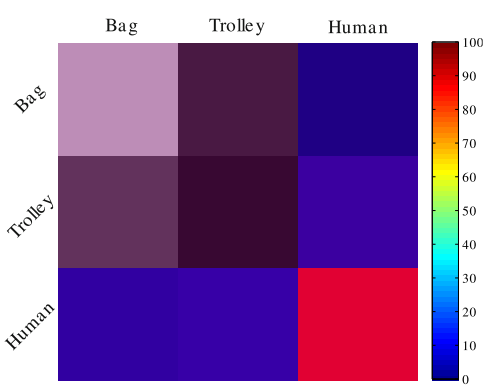

(b)

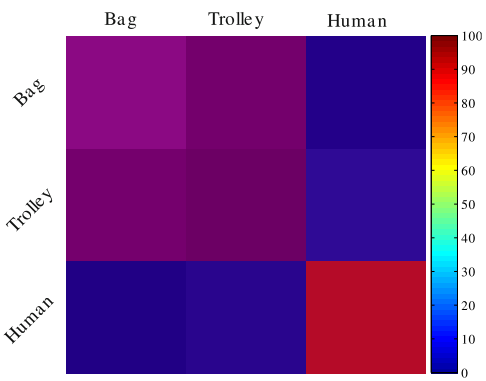

(d)

Fig. 4. Classification Results. (a): Feature selection training curve using relevance analysis. (b): KNN confusion matrix using the 16 most relevant features - testing set accuracy: $82.73 \pm 06.17$. (c): Normalized relevance values of the input feature. (d): SVM confusion matrix using the 16 most relevant features - testing set accuracy: $89.07 \pm 06.69$.

On the other hand, regarding to the attained relevance feature analysis, Fig. 4(c) shows how the described feature selection methodology based on relevance analysis (section 2.3), identifies a decreasing behavior. The relevance curve has a significant fall before the tenth most relevant feature. The above statement can be verified by the obtained classification performance shown in Fig. 4(a), where it is possible to notice how the system performance is reliable before employing the first eight most relevant features. Note that the classification accuracy, and the standard deviation of the system is stable for both classifiers (SVM and KNN), before employing those eight most relevant features. Therefore, the classifiers can achieve success rates with a small subset of features, diminishing the complexity of the problem.

The eight first most relevant features correspond to (1) the percentage of vertical lines, (2) the percentage of diagonal lines, (3) the horizontal standard deviation of the corners, (4) the percentage of vertical lines in the center, (5) the fifth, (6) the sixth, and (7) the third Hu's moments and (8) the bounding box ratio. Lines are the features that best discriminate the objects, with three of them on the top 4. From Fig. 3(b) is evident that the distribution of the lines are well defined in the three objects analyzed, bag and humans have more vertical 
lines in the center than trolleys (blue lines), and trolleys have more diagonal lines (green lines). The Hu's moments also provide a high discriminant power, which proofs the hypothesis that Hu's moments can also describe successfully shapes or contours. Finally, the bounding box ratio depicts the proportion of actual pixels in the object and the total pixels in the region, with humans and bags showing a bigger proportion (see left column in Fig. 3(b)].

The five less relevant features are: the percentage of horizontal lines in the center of the object (being the worst), followed by the fitting ellipse aspect ratio, percentage of horizontal lines in the top, the second $\mathrm{Hu}$ 's moment and the percentage of vertical lines at the right side. Bags, trolleys and humans have horizontal lines in the center and in the top, which explains why these features are part of the less relevant ones. Fitting ellipse aspect ratio is not a good attribute for this classification task, it can be explained because the ellipse fitting can have a similar ratio in the three objects of interest, it would have been better to have included the orientation of the ellipse. The percentage of vertical lines at the right side (weighted at the position 23 in the relevance analysis process ) and also at the left side (weighted at the position 19) are not discriminant for humans and bags, as both are mainly placed in the middle of the bounding box, however, trolley can have more lines in one side depending of their orientation, but in this work this orientation is not considered.

Overall, SVM obtains a better performance than KNN, which can be explained by its generalization capability controlled by the regularization term and the kernel band-width. The presented confusion matrixes in Figs. 4(b) and $4(\mathrm{~d})$ corroborate the latter statement, where is evident how KNN shows higher overlaps among different classes, especially for bags and trolleys.

\section{Conclusions}

In this work, an abandoned object classification methodology to support video surveillance systems was presented, which identifies a set of relevant features to find discriminant patterns. We assumed that the abandoned object was previously detected by a visual surveillance framework, and a preprocessing stage to segment the region of interest from a given object is also presented. Then, some geometric and Hu's moments features are estimated. Given the input feature set, a relevance analysis is employed to identify which features reveal the major variability of the input space to discriminate among different objects samples. Besides, a set of footages from a real-world video surveillance data set was tested. Attained results show how our approach is able to select a subset of relevant features for achieving stable classification performances. Thus, we can conclude that lines are the features that best discriminate our objects of interest. Moreover, the Hu's moments also provided a high discriminant power, successfully describing the object shapes. Therefore, presented approach is able to support the classification stage in automated video surveillance systems, diminishing its complexity while ensuring a stable performance. As future work, we are interested in test our methodology using other kinds of abandoned objects, and we 
also pretend to couple some nonlinear feature extraction methods based on similarity/dissimilarity measures, in order to enhance the classification accuracy. Moreover, it would be interesting to couple the proposed approach with a robust preprocessing stage, in order to provide stable results against noisy scene conditions, such as, light variations, scale changes, occlusions, among others.

Acknowledgments. Research carried out under grants provided by a PhD. scholarship and the project 20201006570 funded by Universidad Nacional de Colombia, and project 20201006594 funded by Universidad Nacional de Colombia and Universidad de Caldas.

\section{References}

1. Silvia, F., Gianluca, G., Carlo, R.: Classification of unattended and stolen objects in video-surveillance system. In: Proceedings of the IEEE International Conference on Video and Signal Based Surveillance, AVSS 2006 (2006)

2. Fan, Q., Pankanti, S.: Modeling of temporarily static objects for robust abandoned object detection in urban surveillance. In: 8th IEEE International Conference on Advanced Video and Signal-Based Surveillance, pp. 36-41 (2011)

3. Kwak, S., Bae, G., Byun, H.: Abandoned luggage detection using a finite state automaton in surveillance video. Opt. Eng. 49 (2010)

4. Otoom, A.F., Gunes, H., Piccardi, M.: Feature extraction techniques for abandoned object classification in video surveillance. In: ICIP, pp. 1368-1371 (2008)

5. Kartoun, U., Stern, H., Edan, Y.: Bag classification using support vector machines. In: Applied Soft Computing Technologies: The Challenge of Complexity Series: Advances in Soft Computing (2006)

6. Huang, Z., Leng, J.: Analysis of hu's moment invariants on image scaling and rotation. In: Computer Engineering and Technology, ICCET 2010 (2010)

7. Daza, G., Arias, J.D., Godino, J.I., Sáenz, N., Osma, V., Castellanos, G.: Dynamic feature extraction: An application to voice pathology detection. Intelligent Automation and Soft Computing (2009)

8. Orozco, J.R., Murillo, S., Álvarez, A.M., Arias, J.D., Delgado, E., Vargas, J.F., Castellanos, G.: Automatic selection of acoustic and non-linear dynamic features in voice. In: INTERSPEECH 2011 (2011)

9. Otoom, A.F., Hatice Gunes, M.P.: Automatic classification of abandoned objects for surveillance of public premises. In: Congress on Image and Signal Processing (2008)

10. Hu, W., Tan, T., Wang, L., Maybank, S.: A survey on visual surveillance of object motion and behaviors. IEEE Transactions on Systems, Man and Cybernetics-Part C, 334-352 (2004)

11. Shotton, J., Blake, A., Cipolla, R.: Contour-based learning for object detection. In: Tenth IEEE International Conference on Computer Vision, ICCV 2005 (2005) 